\title{
ALGUNOS ASPECTOS ESENCIALES DEL PENSAMIENTO DE JEAN WATSON Y SU TEORÍA DE CUIDADOS TRANSPERSONALES
}

\section{SOME ESSENTIAL ASPECTS OF JEAN WATSON THOUGHT AND HER TRANSPERSONAL CARING THEORY}

\author{
Eugenia URra M.* \\ Alejandra Jana A.** \\ Marcela García V. ${ }^{* * *}$
}

\begin{abstract}
RESUMEN
En este trabajo se realiza una síntesis del pensamiento de Jean Watson a partir de dos perspectivas fundamentales a saber: su filosofía de cuidados y su teoría de cuidados transpersonales. El análisis se centra, por una parte, en los fundamentos filosóficos del cuidado que derivan del existencialismo y de la teoría de la alteridad levinasiana y, por otra, en los 10 factores de cuidado que caracterizan su teoría. El artículo además rescata aspectos de la utilización de su teoría de cuidados en diferentes contextos de enfermería. Las autoras del artículo valoran el significado y trascendencia de cada uno de ellos para la disciplina, y la oportunidad que supone aplicarlos en la praxis del trabajo cotidiano y en el desarrollo de una vida buena.
\end{abstract}

Palabras clave: Filosofía en enfermería, Jean Watson, teoría de cuidados transpersonales, enfermería holística.

\begin{abstract}
A display of Jean Watson's thoughts, based on the understanding of her philosophy and theory of transpersonal care is undertaken. On one hand, the analysis is centered in her philosophical assumptions which are based on Existentialism and Levinas theories. On the other hand, the analysis is centered on the ten factors of caring defined on her theory. Beside that this article described the benefits of applying her theory in different contextual nursing situations. The authors make reference to the meaning and transcendence of each one of her ten factors for nursing, and the improvement opportunities coming from their implementation on the way that nurses provide care today as well as on their own personal leaves.
\end{abstract}

Key words: Nursing philosophy, Jean Watson, transpersonal caring theory, holistic nursing.

Fecha recepción: 30/06/11 Fecha aceptación: 29/11/11

\footnotetext{
* Enfermera, docente Departamento de Enfermería, Universidad de La Serena, Chile, Email: eurra@userena.cl

${ }^{* *}$ Enfermera-matrona. Chile. Email: janaacarolina@gmail.com

${ }^{* * *}$ Enfermera atención Primaria. Chile. Email: marcelagarciavera@gmail.com
} 


\section{INTRODUCCIÓN}

Últimamente, en el ámbito de enfermería, se ha publicado una múltiple y variada literatura sobre filosofía, teorías y modelos; quizás porque la disciplina está tomando más fuerza y visibilidad en el tema de los cuidados y su gestión, ejes centrales de los discursos actuales de enfermería. El cuidado es una actividad que concierne a todos los profesionales de la salud, sin embargo enfermería, como profesión sanitaria, es la que destina más esfuerzo, tiempo y dedicación a los cuidados. El cuidado tiene dos dimensiones: una inmersa en la cultura, con sus prácticas individuales y colectivas; y la otra, la moral, que asigna al cuidado un valor, un bien, que legitima el actuar. Es así que las teorías de enfermería que se han desarrollado en las últimas décadas han vislumbrado un desarrollo teórico hacia los cuidados, como lo señala la literatura anglosajona en que destacan los pensamientos de Rogers (1), Leininger(2), Parse(3), Pender(4), Newman(5), entre otras. La disciplina de enfermería debería guiar y orientar las necesidades de la práctica y ésta, a su vez, aplicar o visualizar su utilidad, como también generar teorías inductivas desde la praxis, formándose así un vínculo de retroalimentación, una conexión fundamental mediatizada por la investigación, actividad que genera y crea los conocimientos derivados de una problemática propia de enfermería. Vital es, entonces, entender las nuevas concepciones teóricas con una visión de cuidados humanos, que puedan movilizar la enseñanza y práctica tradicional de enfermería, la que muchas enfermeras sostienen desde el paradigma de la totalidad, centralizada en una ciencia biomédica y concepciones de enfermedad, tecnologías, y un marcado lenguaje medicalizado, hacia otro paradigma: el de la simultaneidad, que se centraliza en lo que Rogers (6) enuncia como la ciencia de los seres unitarios para enfermería, es decir una ciencia humana.
El comprender los fundamentos ontológicos del cuidado y sus componentes teóricos desarrollados por estas teoristas permite, claramente, dar un sentido coherente y consistente a enfermería como una ciencia humana. Por esto la teoría de Jean Watson es útil, ya que permite plantear una filosofía de cuidados, un lenguaje teórico propio y una relación entre teoría-práctica que revitaliza aspectos perdidos o invisibilizados de la enfermería como los que plantea Sarter (7) en su revisión de las fuentes filosóficas de la teoría de Watson: el alma (espíritu, o interior de sí), el dualismo (experiencias subjetivas/objetivas), la armonía, la casualidad y el tiempo, la evolución espiritual, y la autotrascendencia, aspectos que han estado siempre presentes en enfermería pero que han sido opacados o subvalorados por el enfoque positivista y tecnológico. La teoría de Jean Watson, de corte existencialista, humanista y con una profunda influencia de la teoría de la alteridad levinasiana, es un ejemplo que da cuenta de la necesidad de rescatar los elementos fundantes del cuidado que en último término permitirán su trascendencia.

Margaret Jean Watson, nació en los Estados Unidos de Norteamérica en 1940, es licenciada en enfermería con una maestría en enfermería en salud mental y psiquiatría, y posteriormente realiza un doctorado en psicología de la educación y consejería. En 1979 Watson publica su primer libro: Enfermeria: The philosophy and science of caring (8) (La filosofía y ciencia de los cuidados), en el que expone su teoría, la que en sus palabras: "emergió desde mi inquietud por otorgar un nuevo significado y dignidad al mundo de la enfermería y al cuidado de los pacientes".

Posteriormente, Watson corregirá y complementará el sentido original de su teoría y estructura de los diez Factores Caritativos (FC) por el de Proceso Caritas o Proceso de Cuidar (PC) que fueron expuestos en su libro: Nursing: Human science and human care. A theory of nursing (9) (Enfermeria: ciencia y cuidados humanos. Una teoría de Enfermería) 
en 1985, reimpreso en los años 1988 y 1999 (10). En este libro afirma que expande los aspectos filosóficos y transpersonales del " $m o$ mento de cuidados" como el eje de su marco de referencia y hace más evidente los aspectos éticos, del arte y los espirituales-metafísicos. Además, impregnada en el pensamiento postmoderno, Watson publica su libro: Postmodern nursing and beyond (Enfermería posmoderna y otros enfoques) (11) en el año 1999, que se basa en la ontología de las relaciones y fundamento ético-ontológico frente a una epistemología de la ciencia y tecnología. En este libro se desarrollan los aspectos espirituales y energéticos de los cuidados, conscientes e intencionales, más la presencia y evolución humana de las practicantes.

Su trabajo más reciente es: Caring science as sacred Science (12) (Ciencia del cuidado como Ciencia sagrada), libro que, según la autora, invita a reflexionar sobre el fundamento moral y metafísico de la conducta profesional de enfermería. Watson(9) también afirma que se ha apoyado en la filosofía de la alteridad de Emmanuel Levinás y Knud Logstrud en la búsqueda de un modelo de ciencia que reintegre la metafísica con el dominio material y así reencuentre la trascendencia en el ser en un mundo con los otros. Actualmente, Watson se desempeña como académica en la Escuela de Enfermería de la Universidad de Colorado y es una de las fundadoras del Centro de Cuidados Humanos, que desarrolla el conocimiento del cuidado en la sanación de los individuos con un aporte de una base científica y moral para la práctica clínica.

Watson reconoce que el cuidar es parte fundamental del ser y es el acto más primitivo que un ser humano realiza para efectivamente llegar a ser, este ser es un ser en relación a otro que lo invoca. Este llamado desde el otro, absolutamente otro, es la base fundadora del cuidado en general y, a la vez, del cuidado profesional propio de la enfermería. Este cuidado profesional es estructurado, formalizado y destinado a satisfacer las necesidades del ser humano con el propósito de promover, mantener o recuperar la salud (12). Si bien todo cuidado responde a una necesidad, el cuidado sanitario tiene metas específicas, se apoya en un contexto epistemológico, y se formaliza y desarrolla por medio de una serie de técnicas aprendidas en la formación profesional de enfermería. Por lo expuesto, es que este artículo se centralizará en el pensamiento y evolución de la teoría de Watson, los postulados centrales de su teoría y su aplicación en diferentes contextos. De este modo, las autoras desafían a los lectores a repensar la disciplina y la praxis, como parte sentida y valorada, desde las ciencias humanas de los cuidados en nuestra comunidad de enfermería, lo que implica una transformación de nuestro ser y nuestras prácticas.

Los cuidados en nuestra cultura y tiempos históricos actuales necesitan una fuerte reconsideración hacia la humanización de la salud, en la que los cuidados se sitúan ética y profesionalmente, pero que están en una tensión con los discursos dominantes, a saber: la tecnología, la enfermedad y la práctica basada en la evidencia; visiones en donde la enfermería y sus practicantes no aclaran aún por cuál corriente optar o cómo intentar convivir con ellas.

Nos situamos además en un mundo globalizado, pluricultural y secular donde la negación del otro se ha transformado en una práctica cotidiana. Las enfermeras/os no estamos ajenas/os a ello, es por esta razón que teoristas como Watson son vigentes y oportunas para que podamos reflexionar y repensar nuestras formas de conducirnos en el trabajo diario. Esperamos que este artículo ayude a iluminar sobre la importancia trascendente de los cuidados y la necesidad de un cambio paradigmático, una apertura a lo posible, basándonos en Watson y su trabajo meticuloso, profundo y acabado sobre lo que los cuidados significan, tanto para la humanización de la praxis de enfermería como para su colectivo profesional. 


\section{UNA VISIÓN DE LA FILOSOFÍA Y TEORÍA DE WATSON}

Jean Watson basa la confección de su teoría en siete supuestos y diez factores de cuidados o factores caritativos de cuidados (FC), los que serán examinados. En primer lugar, los supuestos, que son premisas asumidas como verdad sin comprobación, se detallan a continuación (13):

- El cuidado sólo puede ser demostrado y practicado efectivamente en una relación interpersonal, es decir, el cuidado se transmite de acuerdo a las prácticas culturales que tienen las distintas comunidades; y en forma particular, en enfermería de acuerdo a las necesidades humanas, sociales e institucionales. Los cuidados son contextuales a la época, al lugar geográfico, a las necesidades de una población o de un individuo en particular, y a los elementos con que se cuenta y por ello requieren de un conocimiento del ambiente que rodea al individuo y del conocimiento del individuo en sí.

- El cuidado está condicionado a factores de cuidado, destinados a satisfacer necesidades humanas. El cuidado tiene destino, efectos y objetivos.

- El cuidado efectivo promueve la salud y crecimiento personal y familiar. El cuidado promueve la satisfacción de necesidades, por tanto propende a la relación armónica del individuo consigo mismo y su ambiente.

- Un ambiente de cuidado es aquel que promueve el desarrollo del potencial que permite al individuo elegir la mejor opción, para él o ella, en un momento preciso.

- La ciencia del cuidado es complementaria de la ciencia curativa. El cuidado no es sólo curar o medicar, es integrar el conocimiento biomédico o biofísico con el comportamiento humano para generar, promover, o recuperar la salud.
- La práctica del cuidado es central en la enfermería. Un individuo debe ser acompañado en las fases de toma de decisiones y no sólo ser un receptáculo de información. El acompañar es necesario para que, paulatinamente, el individuo descubra cómo, desde una situación particular, puede encontrar la mejor solución desde su propia realidad. Enfermería, entonces, debe focalizarse en un acompañar sensible pero responsable, basado en el conocimiento y en la práctica de una actitud terapéutica.

En segundo lugar expondremos los diez FC que después Watson (14) denomina Proceso Caritas de Cuidados (PCC), que corresponde a una extensión de los anteriores, pero ofrece un lenguaje más fluido para entender en un nivel más profundo las dimensiones del proceso de vida de las experiencias humanas. Así se expondrá una síntesis de los originales FC y su transformación luego al PCC, puntualizando sobre su marco conceptual para facilitar la comprensión de los cuidados de enfermería como la ciencia del cuidar. Estos son (13):

\section{1. "Formación humanista-altruista en un sis-} tema de valores", (8) se convierte luego en la "práctica de amorosa bondad y ecuanimidad en el contexto de un cuidado consciente". Watson asume que el "día a día" de la práctica profesional requiere que la enfermera/o evolucione en su desarrollo moral. Si bien la formación de cada uno en la infancia y juventud temprana está marcada por el contexto socio-cultural de pertenencia, esto no significa la permanencia en un sistema estático. Es importante una acabada reflexión sobre el propio desarrollo moral, ya que es la única forma de comprender los sistemas morales ajenos. Esto parte de una reflexión de la propia experiencia y del desarrollo a lograr individualmente. De esta forma la introspección debería formar parte de nuestras prácticas diarias, tal como lo constituye, por ejemplo, 
la meditación para otras culturas. Es útil así la meditación, la reflexión y la terapia en caso necesario, así como el desarrollo de aptitudes artísticas que ayuden al profesional a encontrarse consigo mismo.

Este sistema de valores debe combinarse con el conocimiento científico que guía la acción de la enfermera, pero sin olvidar que los conocimientos científicos por sí solos no ayudarán a la relación con otros. Por esto, un paciente no es un personaje sobre el cual es factible escribir en primera persona, es un ser humano libre que debe narrar su propia biografía.

Watson otorga gran importancia a la formación en ciencias humanas, aspecto muy olvidado en la formación profesional, que brinde las herramientas necesarias para comprender las dinámicas sociales, culturales y psicológicas de los individuos. No es posible elegir sólo cuidar a aquellos que concuerdan con nuestro sistema de valores, raza, clase social, generación, etc. Cuidamos a seres humanos diferentes y únicos. Watson por tanto, integra -emulando a Gilligan (15)- el cuidado amoroso con la ecuanimidad, esto requiere de un desarrollo evolutivo moral, no sólo de un desarrollo del conocimiento.

2. “Incorporación de la fe - esperanza", se convierte luego en "Ser auténticamente presente $y$ permitir y mantener el sistema de creencias profundas y subjetivas del individuo, compatible con su libertad. Este individuo libre, con creencias propias, es un ser para el cuidado". Este factor habla de la importancia de la fe y la esperanza para el cuidado y la sanación, dado que ha estado presente a lo largo de toda nuestra historia y por lo tanto también a lo largo de la narrativa que da cuenta de nuestra convivencia como especie humana, en los que ha habido influencias de lo mágico, las oraciones y los encantos. De este modo, el permitir que los individuos cultiven su sistema de creencias y ejecuten sus rituales para permitir que les ayude a mantener la fe en ellos mismos, contribuirá a la sanación o mantención de la salud. Esto proviene de una costumbre arcaica, ya que en muchas culturas la fe y la sanación eran resorte del mismo oficiante; sucede así, por ejemplo, en las comunidades indígenas, y así es como actualmente se intenta incorporar sus propios rituales. Particularmente en Chile, en la asistencia del parto de miembros de la comunidad Aymará, o en EE.UU, donde a los miembros ortodoxos de la comunidad judía se les permiten los alimentos kosher. Entonces, y de acuerdo al viejo aforismo, "no siempre es factible curar, pero siempre cuidar", la fe no necesariamente logrará la curación; pero siempre estará asociada al cuidado.

3. "El cultivo de la sensibilidad hacia uno mismo y hacia los otros" (8) será redactado más tarde como "El cultivo libre de las propias prácticas espirituales y transpersonales, que vaya más allá del ego y la apertura a los demás con sensibilidad y compasión". Para Watson el ser humano es en primer lugar un ser sintiente. Es habitual que en la práctica sanitaria se separe el desarrollo profesional de este ámbito, producto de lo cual las personas tienden a esconder, negar o disimular sus sentimientos. Esto llevará a no tratar con los propios sentimientos, a temerles y a no poder cambiarlos cuando éstos no son positivos. Un individuo que rehúsa reconocer sus propios sentimientos, difícilmente logrará entender los de los otros. Por otra parte, se tiende a proteger al paciente de sentimientos negativos, para que no llore, o muestre ansiedad. Estos sentimientos forman parte de la vida y de los procesos que vivimos como seres humanos.

Un ejemplo: $i$ Es prudente evitar que una madre mire el rostro de su hijo que ha nacido muerto? Con esta práctica sólo aumentará el dolor, y negará la evidencia que iniciará el duelo. Esta práctica se apropia del dolor de otro, impide su libre expresión por desgarradora que sea. El duelo de esta madre es personal, forma parte de su vida y no le atañe al personal sanitario evitarlo. En estas oca- 
siones el silencio, el respeto y un abrazo son más útiles; y por cierto, más humanos. Por tanto la expresión de los sentimientos es una forma de empatía con otros que trasciende el propio yo.

Las conductas de evitación también tienden a ser habituales, como el temor a enfrentarse al paciente que va a morir, la aprensión a contestar preguntas, y enfrentar situaciones de duelo y dolor. Todas estas situaciones están presentes en el día a día de la labor de enfermería. Es por ello que el practicar la sensibilidad hacia sí mismo y los otros adquiere relevancia, y Watson destaca que esta sensibilidad se ve reforzada por la formación valórica. El cultivar un desarrollo evolutivo moral debe ser tan importante como mantenerse al día en las técnicas y conocimientos, como asimismo la apertura a desarrollar sentimientos como la fe y esperanza. El desarrollo de la sensibilidad forma parte de un ser auténtico y honesto, que no teme develar su rostro ante la vulnerabilidad y el dolor (8).

4. "El desarrollo de una relación de ayuda y confianza" (8). El cuidar la relación humana se convierte en "Desarrollo y mantenimiento de una auténtica relación de cuidado, a través de una relación de confianza". Una relación de cuidado depende de los factores de cuidados enunciados anteriormente. Esta relación de confianza va unida con la promoción y aceptación de sentimientos positivos y negativos. Para esto es necesario la sensibilidad, la apertura y el altruismo, pero también la congruencia, que tiene que ver con la honestidad, con la percepción de realidad y con el apego a la verdad. Watson delimita tres guías para evitar la incongruencia: en primer término, las enfermeras/os no deben jugar roles estereotipados, ni emitir discursos programados cual "contestador telefónico", ya que esto bloquea la autenticidad y aleja al paciente; en segundo término, el ser capaz de reconocer las dificultades que conlleva la relación con otros pasa por el comprender que toda relación, al menos, tiene dos emisores y dos re- ceptores; y en último término, que si bien las experiencias que otorgan los pacientes son vitales, también lo son las propias experiencias. Este es un proceso de autoaprendizaje, en el que la introspección es fundamental.

La relación de cuidado está basada en la creencia que el ser para el cuidado es único, es persona y es capaz de ejercer su libertad y su autonomía, en mayor o menor grado. Más específicamente, los pacientes o usuarios no nos pertenecen, ni su cuerpo, ni sus problemas, ni su espíritu. A modo de ejemplo: muchas veces en el cuidado de un paciente grave, que no está consciente y no puede ejercer autonomía en relación a sus funciones corporales, la certeza que es un cuerpo ajeno, pero el cuerpo de una persona, ayudará a no cosificarlo y a respetarlo como tal.

5. "La aceptación de expresiones de sentimientos positivos y negativos" (8). Esta es una parte inherente a la formación de una relación de ayuda y de confianza. Watson la enuncia más tarde como un "estar presente y constituirse en apoyador de la expresión de los sentimientos positivos y negativos en una conexión con la espiritualidad más profunda del ser que se cuida". Las emociones juegan un rol preponderante en la conducta humana y para Watson son "una ventana por la cual se visualiza el alma". Es ampliamente aceptado que la comprensión intelectual y la emocional de la misma información son bastante diferentes, esto es más acentuado cuando las situaciones conllevan un efecto negativo, por ejemplo, situaciones como la ansiedad, el estrés y la confusión, o incluso el temor, pueden alterar la comprensión y la conducta. La libre expresión de estos sentimientos permite no elaborar sentimientos defensivos, de negación o aumentar el estrés. La labor del profesional de enfermería es permitir la expresión de estos sentimientos, que es congruente con visualizar al otro como un ser para el cuidado.

6. "El uso sistemático de una resolución creativa de problemas del proceso asistencial" (8) se 
convierte en: "el uso creativo de uno mismo, como partícipe en el arte de cuidar y de todas las maneras de conocer como parte del proceso asistencia". Watson se refiere a la sistematización excesiva de normativas que impiden el desarrollo creativo de esta profesión. Las enfermeras realizan multiplicidad de procedimientos y suponen que la corrección de estos procedimientos es la manifestación de la utilización de un método científico, por ejemplo, el uso del proceso de enfermería, el que Watson visualiza como un método de resolución de problemas para asistir con una decisión a todas las situaciones de enfermería. Esto es importante, porque la sistematización de los procesos no debe ser un impedimento para investigar, estudiar y desarrollar habilidades, para enfrentar situaciones nuevas. El mal uso de los formatos impide visualizar la profundidad del fondo, cuando se realizan mecánicamente, esto es relevante pues -en último término- es lo que distingue a un técnico de un profesional. El proceso de enfermería - un instrumento que aún se utiliza y que está abocado a diagnosticar las necesidades de los pacientes y a programar las acciones para darles solución, como asimismo a la evaluación de estas accionesse suele convertir en un proceso mecánico, que normalmente no está centrado en el paciente sino en la patología. Si se agrega a esto un deficiente profesionalismo, en el sentido de no ser reconocidos por el colectivo social como auténticos profesionales y con autopercepción de sacrificio y sumisión, puede desembocar en una posición rígida, autocontrolada, y autodestructiva, lo que daña profundamente al propio practicante y dificulta la relación terapéutica. El no delimitar el campo propio de realización de enfermería y referirlo a un campo biomédico no hacen posible la autonomía y la creatividad.

Según Watson, para cumplir la misión de brindar cuidados efectivos es indispensable cambiar esta imagen, adquirir autoconfianza y ejercer más autonomía. Para esto el instrumento privilegiado es la investigación, el aprendizaje, la apertura a nuevas estrategias de cuidados, establecer correcciones a las teorías existentes y elaborar nuevas. En resumen, es vital desarrollar un marco de conocimientos respecto al cuidado.

7. "La promoción de una enseñanza-aprendizaje interpersonal" (8). Luego deriva a: "Participar de una verdadera enseñanza-aprendizaje que atienda a la unidad del ser y de su sentido y que trate de mantenerse en el marco referencial del otro". Este factor de cuidado es el que más ayuda al usuario a ser partícipe activo del estado de su propia salud y a tomar las decisiones en pos de mantenerla, recuperarla, mejorarla, o en otros casos a prepararse para determinados eventos. La enfermería como profesión ha estado muy ligada a la educación en salud; sin embargo, Watson considera que la educación que se brinda es ante todo información, concretizada en una lista de acciones a realizar o a evitar. Dicha información no se apropia de un sello que la distinga de otros modos de obtenerla, como por ejemplo a través de los medios de comunicación, donde también se brinda educación en salud, periódicamente. La educación en salud, efectuada por profesionales de la salud, requiere establecer una relación intersubjetiva para otorgar una educación personalizada y dirigida. La educación en salud persigue un cambio positivo y proactivo de los usuarios, cambio que -según Watson- es difícil de obtener, si no se incorporan nuevos paradigmas. La información -prosigue Watson- es un componente de la educación, pero no el todo. Si bien se han dado importantes pasos en este rubro, no es menos cierto que por escasez de tiempo, o motivación, se suele seguir informando a los pacientes, sin educarlos, y sin educarse a través de ellos, ya que la educación es un proceso de retroalimentación para ambas partes, y no una mera entrega de conocimientos.

El cuidado supone el brindarlo en base a las necesidades sentidas de los usuarios/pacientes/clientes. Una actitud efectiva de cui- 
dado, por tanto, supone un conocimiento de lo que los individuos necesitan. Personalizar los cuidados es, en buenas cuentas, mantener una actitud respetuosa hacia las particularidades de un individuo, comunidad o población. La generalización tiende a neutralizar los sujetos, a masificarlos y, además, a no obtener buenos resultados.

Según Rogers -a quien cita Watson- la enseñanza no es función de un buen currículum clínico ni académico del educador, es un proceso mucho más complejo, que parte de tener la habilidad para lograr, en el marco de una relación -en este caso, educador-educando- cambios de conducta. Esto pasa necesariamente por nutrirse de los educandos, y no de un mero recitar conocimientos.

8. "La creación de un entorno protector y/o correctivo para el medio físico, mental, espiritual y sociocultural" (8) se convierte en: "creación de un medio ambiente de cuidado en todos los niveles (físico, no físico, ambiental de energía $y$ de conciencia) ya sea el todo, belleza, confort, dignidad y paz". Watson divide este ambiente en externo e interno, los cuales son interdependientes entre sí. Ambos contienen variables, por ejemplo del medio ambiente externo surgen las variables como seguridad, confort, abrigo; y del medio ambiente interno surgen la autorrealización, comunicación, etc. A partir de estas variables surgen las necesidades, que Watson define como el requerimiento de una persona, que de ser satisfecho, alivia o disminuye su angustia o dolor, o mejora su sentido de adecuación y bienestar. La satisfacción de estas necesidades tiene estrecha relación con la salud del individuo.

9. "La asistencia con la gratificación de necesidades humanas" (8). Las que posteriormente llama: "La asistencia en necesidades básicas, con un cuidado consciente e intencional que toca y envuelve el espíritu de los individuos, honrando la unidad del Ser, y permitiendo a la espiritualidad emerger". Las necesidades humanas están estratificadas. De acuerdo a Watson, el primer nivel está representado por las necesidades de supervivencia o necesidades biofísicas, por ejemplo: nutrición, eliminación y ventilación. En el segundo nivel se ubican las necesidades funcionales o psicofísicas como reposo - actividad, y las sexuales. En un tercer nivel, las integrativas o necesidades psicosociales, de pertenencia y logros. En el nivel más elevado se ubican las necesidades intra/interpersonales; la necesidad de realización del sí mismo, tendientes a la búsqueda de crecimiento personal.

Watson acota que los factores socioculturales afectan todas las necesidades en todos los niveles. El brindar cuidados es, por tanto, el trabajo profesional destinado a la satisfacción de necesidades humanas en el ámbito de la salud de un individuo, comunidad o población, como también integrativo y holístico, porque abarca los diferentes estratos de necesidades.

10. "El reconocimiento de fuerzas fenomenológicas y existenciales" (8). Este último factor de cuidado es el método para lograr trabajar en la satisfacción de ellas: la fenomenología, concepto que Watson define así: "se refiere al énfasis en la comprensión de las personas de cómo las cosas aparecen ante ellos... La fenomenología es descriptiva, es una descripción de datos que aparecen en una situación dada y ayuda a entender el fenómeno en cuestión (10). Esta orientación es útil para la enfermera, ya que ayuda a reconciliar o mediar la incongruencia de los diferentes puntos de vista de la persona en forma holística; y al mismo tiempo, atender al orden jerárquico de sus necesidades" (16).

Los diez factores expuestos por Watson ayudan a delimitar lo que es el cuidado profesional, ya que si bien una enfermera/o, puede ayudar al otro a resolver problemas y promover a restaurar su salud, no puede crearle ni restaurarle la salud. La enfermera, entonces, como acota Watson, es responsable 
de ofrecer condiciones para que el paciente/ usuario resuelva sus propios predicamentos. Cada persona desarrolla su forma de encontrar significados en la vida, y esto es válido para los individuos enfermeras/os, ya que confrontan su forma de estar en el mundo ante un evento que es la enfermedad, pero éste es un proceso individual, en toda su profundidad.

\section{APLICACIÓN DE LA TEORÍA DE J. WATSON}

La teoría de Watson ha tomado fuerza en diferentes lugares del mundo a través de un Instituto y Consorcio Internacional de Cuidados Caritas. Actualmente, la teoría de Watson tiene una conexión con 29 países, lo que refleja la trascendencia de ella. También existe una variada literatura que ha expuesto la utilidad de la teoría de Watson en la práctica. Por ejemplo, Bent et al. (17) consideraron la conceptualización de Watson en un sistema de salud en Colorado, Estados Unidos, como modelo guía de la práctica, basados en la relación de personas, sistemas y experiencias. Dentro de las partes del proyecto, la unidad Nightingale creó un ambiente propicio para cambios con el personal usando el Reiki, la Reflexología, la pintura, la decoración de la unidad y el uso de la música. La teoría de Watson fue una guía iluminadora que sostuvo la fundamentación y apoyó la práctica dado por su beneficio en cultivar una conciencia de cuidados, y el establecimiento de una relación terapéutica fuerte y sostenible.

En Canadá, Cara (18) en una educación continua instó a las enfermeras a aplicar la teoría de Watson en su práctica clínica ya que frente a la deshumanización en los sistemas sanitarios, las enfermeras deben hacer un esfuerzo consciente por preservar el cuidado. Rafael-Falk (19) ha extrapolado la teoría de Watson usando los conceptos de holismo, atención y ecología, para afirmar que es con- gruente con los enfoques contemporáneos de salud de la comunidad en la atención primaria, la promoción de la salud, y en modelos de desarrollo de la comunidad.

En Brasil, Do Nascimento y Lorenzini (20), usando el enfoque de la investigaciónacción para recolectar una variedad de datos de las acciones de cuidados realizadas, dan cuenta de un proyecto cualitativo situado en un hospital de Santa Caterina. Este grupo implementó este proyecto en familias y pacientes de una unidad de emergencia, visto como una experiencia innovadora y desafiante de autoconocimiento y humanización de las relaciones de cuidado. En el mismo país, Sueuda et al. (21) evaluaron los conceptos teóricos de la teoría de Watson realizando un cruce, entre la percepción de pacientes portadores de lesión medular del tipo paraplejia y las enfermeras que interactuaban con esos hospitalizados. Dentro de su evaluación, los autores señalan: "teóricamente las enfermeras... asumían ese compromiso [del cuidado humano como el ícono de la teoría, de la práctica, y de la investigación]; sin embargo, en la práctica lo distorsionaban en nombre de la neutralidad científica" (21).

En España, Velásquez et al. (22) incorporaron el concepto de sufrimiento espiritual dentro de un plan de cuidados en el cual asignaron importancia medular al acto de rezar, como parte de los efectos positivos en la salud de las personas y a la teoría de Watson incorporada en la extensión de vivencias espirituales.

En Chile ha habido intentos de búsqueda para su comprensión como en Ayala et al. (23), quienes mostraron el proceso de traducción y pilotaje del Caring Behaviors Assessment Tool al castellano para posterior validación. Poblete (24), recientemente, en su tesis doctoral usó la teoría de Watson en la percepción del cuidado en enfermeras asistenciales y académicas.

Los comportamientos y conductas no son fáciles de cambiar abruptamente, tanto en las personas como en las instituciones, quienes 
culturalmente, por largo tiempo han privilegiado formas de cuidar basada en la racionalidad empírica y la tecnología médica que la refuerza (25). Enfermería debe entender que ha seguido más a la ciencia médica $y$, por ende, a la enfermedad y al curar. Aunque seguiremos conviviendo con ellas, las enfermeras/os deben empezar a valorar los cuidados como algo mucho más apreciado que la complementariedad a la medicina, al otorgarle representación y visibilización en la práctica, siendo así sus mismas practicantes y sus usuarios los beneficiados.

\section{CONSIDERACIONES FINALES}

Este artículo ofrece una interpretación de la obra teórica de Watson, que se basa en concepciones filosóficas existenciales y fenomenológicas, pero que también da cuenta de una influencia espiritual y ecológica, recogidas de la filosofía oriental Hinduista (26). Watson intenta, a lo largo de su trabajo, fundamentar el cuidado como una forma de habitar en el mundo, en un mundo que no nos es ajeno, mundo al que hemos venido para desarrollar un proyecto, que nos permitirá trascender y cuya expresión más primitiva es el cuidado genuino, un mundo con los otros donde somos invocados e interpelados, desde la desnudez propia de la vulnerabilidad humana (27), aspecto que aporta y da contenido ético a nuestro proyecto de corte existencialista.

El cuidado, expuesto en forma de supuestos y factores, da cuenta de una metodología inspirada en la fenomenología como una forma de aproximación para que el cuidador profesional se convierta en instrumento terapéutico y por último, y no menos importante, una forma de entender la relación intersubjetiva, interpersonal y transpersonal situada en una esfera cósmica, donde cada acción tiene un efecto, lo que además impone una responsabilidad, entendida como una respuesta de profundo contenido ético en la relación con usuarios o clientes, y estimulando un proceso de individuación dinámico y coherente con el convivir (28).

Hemos intentado, además, exponer alguna literatura en diferentes contextos de enfermería, ya que actualmente hay varios hospitales en que enfermeras y líderes en la administración, utilizan y aplican las bases filosóficas, teóricas y morales de Watson (29). Esperamos así que estos ejemplos inspiren y contagien la apertura de re-pensar y hacer enfermería, realizando una síntesis entre teoría y práctica actualizada y vigente. Fue nuestro deseo, también, mostrar la teoría de Watson para que despierte al quehacer académico, ya que este trabajo da cuenta de una reflexión profunda: que enfermería, en tanto humano, precisa del convivir en un mundo con los otros, mostrando la necesidad de incluir las ciencias humanas en nuestra formación de pre y postgrado.

Queremos terminar con una frase de Jean Watson, que inspiró la confección de este artículo: "Nursing has lost its soul to medical and technical and evidence-based practices, which we need", Watson agrega; "But without the human dimension, it can be destructive to human beings. It's foundational to sustaining humanity and putting caring back into our systems"1 (30).

\section{REFERENCIAS}

1. Rogers ME. An introduction to the theoretical basis of nursing. Philadelphia: Saunders; 1970.

2. Leininger MM. Caring: an essential human need. Detroit: Slack; 1991.

\footnotetext{
1 Traducción de las autoras: "Enfermería ha perdido su alma por servir ya sea a la la medicina, la tecnología y la práctica basada en la evidencia, las que por cierto se necesitan; pero sin la dimensión humana, esto puede ser destructivo. Es fundamental preservar la esencia humana y reposicionar el cuidado dentro de nuestros sistemas" [sanitarios].
} 
3. Parse RR. Man-living-health: A theory of nursing. New York: Wiley; 1981.

4. Pender N. Health promotion in nursing practice. New York: Appleton-Century Crofts; 1982.

5. Newman MA. Health as expanding consciousness. St. Louis: Mosby; 1986.

6. Rogers ME. Nursing science and art: A prospective. Nurs Sc Q. 1988; 1(3): 99102.

7. Sarter B. Philosophical sources of nursing theory. En Reed PG, Shearer N, Nicoll L. Perspectives of nursing theories. 4 ed. USA: Lippicontt Williams \& Williams. 2004; cap. 32.

8. Watson J. Nursing: The philosophy and science of caring. Boston: Little Brown; 1979.

9. Watson J. Nursing: Human science and human care. Connnecticut: AppletonCentury Croft; 1985.

10. Watson, J. The theory of human caring: retrospective and prospective. Nurs Sc Quart. 1997: 10(1): 49-52.

11. Watson J. Postmodern nursing and beyond. New York: Harcourt-brace/Elsevier; 1999.

12. Watson J. Caring science as sacred Science. Philadelphia: FA Davis; 2005.

13. Watson J. Theory evolution: Watson's caring theory [Internet]. Colorado (USA): University of Colorado Health Science; 2006 [citado 11 mayo 2011]. Disponible en http//: www.ucdenver.edu/.../caring/ humancaring/.../HumanCaring

14. Watson J. Watson theory of human caring and subjetive living experiences: Carative factors/caritas process as a disciplinary guide to the professional nursing practice. Text Cont Enf. 2007; 16(1): 129-135.

15. Gilligan C. In a different voice: Psychological theory and Women's development. Harvard University Press; 1982. pp. 5-23.

16. Talento B. Watson's theory. Julia B George (ed.) Nursing Theories. Connecticut (USA): Appleton-Lange; 1991.
17. Bent K, Burke JA, Eckman A, Hottmann T, McCabe J, Williams RN. Being and creating caring change in a health care system [Internet]; 2003 [citado 10 mayo 2011]. Disponible en htpp//www. watsoncaringscience.org/pdf/BeingandCreatingCaring.pdf

18. Cara C. The pragmatic view of Jean Watson's caring theory. Int J Hum Caring 2003; 7(3): 51-51.

19. Rafael-Falk AR. Watson's Philosophy, Science, and Theory of Human. Caring as a Conceptual Framework for Guiding Community Health Nursing Practice. ANS 2000; 23(2): 34-49.

20. Do Nascimento C, Lorenzini A. Cuidado transpessoal de enfermagem a seres humanos em unidade critica. R Enf UERI 2006; 14(3): 333-41.

21. Sueuda CM, De Oliviera M, Da Silva MJ. Evaluación de la aplicabilidad de la teoría de Watson en un estudio empírico. Enf Integral 2007; 20(4): 499-503.

22. Velásquez MP, Rebolledo D, González J. Cuidados de enfermería en el sufrimiento espiritual aplicando el modelo de Jane Watson. Salud, Arte Cuid 2009; 2(2): 8-13.

23. Ayala R, Calvo J, Torres MC, Koch T. Evidencias para la filosofía de Watson: versión preliminar del caring behavior assessment in Chile. Rev Cubana Enf. 2010; 26(1): 42-51.

24. Poblete M. Cuidado humanizado: percepción de autoeficacia en enfermeras académicas y asistenciales. [Tesis Doctoral]. [Concepción, Chile]: Universidad de Concepcion; 2009. 145 p.

25. Watson J. Nursing's caring -healing paradigm as exemplar for alternative medicine? Alter Therap 1997; 1(3): 64-69.

26. Watson J. Nursing. Human science and human care. A theory of nursing. USA: Jones \& Bartlett Pub; 2007.

27. Lévinas E. Ética e infinito. Madrid: Editorial Visor; 1991.

28. Watson J, Smith M. Caring science and 
the science of unitary human being: a trans-theoretical discourse for nursing knowledge development. J Adv Nurs 2002; 37(5): 452-461.

29. Watson J. Caring science and human caring theory: transforming personal and professional practices of nursing and health care. JHHSA (Spring), 2009.

30. Watson J. Global Effort: Caring theory put back into practice around the globe [Internet]. [citado 2 junio 2011]. Disponible en: http://www.denvernursingstar.com/newsletter/newsletter_view. asp? newsid $=3472 \&$ mode $=$ current 